\title{
Generalized Proportional Allocation Mechanism Design for Multi-rate Multicast Service on the Internet
}

\author{
Abhinav Sinha and Achilleas Anastasopoulos
}

\begin{abstract}
In this paper we construct two mechanisms that fully implement social welfare maximising allocation in Nash equilibria for the case of single infinitely divisible good being demanded by a separate groups of agents, whilst being subject to multiple inequality constraints. The nature of the good demanded is such that it can be duplicated locally at no cost. The first mechanism achieves weak budget balance, while the second is an extension of the first, and achieves strong budget balance at equilibrium. One important application of these mechanisms is the multi-rate multicast service on the Internet where a network operator wishes to allocate rates among strategic agents, who are segregated in groups based on the content they demand (while their demanded rates could be different), in such a way that maximises overall user satisfaction while respecting capacity constraints on every link in the network. The emphasis of this work is on full implementation, which means that all Nash equilibria of the induced game result in the optimal allocations of the centralized allocation problem.
\end{abstract}

\section{Introduction}

Allocation of services on the Internet is an important problem, not only from the system performance point of view but also from an economic standpoint. As requirements from Internet services increase, having appropriate markets for various services could lead to more efficient use of available resources.

Once one introduces the notion of allocation of resources based on requiring specific payments for services provided, the next logical step would be to model the system agents as being strategic i.e. utility-maximising. In a general informationally (and physically) decentralised system, such as the Internet, in order to make protocols work the designer would usually require dissemination of (local) information from agents. It is here that enforcing of protocols becomes harder when strategic users are present. For example, if a protocol requires agents to take action based in the interest of whole network then in the absence of true local information, the designer cannot check whether an agent adheres to the protocol. For strategic agents, the one thing we can ensure is

Key words and phrases. Multicast routing, propotional allocation, game theory, mechanism design, full implementation, Nash equilibrium. 
that actions taken by agents would be so as to maximise their own profit. So the decentralised information structure along with strategic agents requires the designer to venture into the field of mechanism design. Mechanism design focuses on designing contracts which enforce strategic agents to take actions that reveal their private information truthfully. Voting rules, auctions, private and public good exchange economies are a few examples of fields where Economists have used mechanism design extensively.

In this paper, we focus on a specific aspect of mechanism design, Nash Implementation. Without going into a formal definition, Nash implementation refers to design of contracts such that only the designer's most preferred outcome is realised as a result of interaction between strategic agents (Nash equilibria), whereas in general mechanism design, other less preferred outcomes are also possible. Thus implementation is more stringent and is capable of producing better allocations. For implementation, readers may refers to survey article [1] where the discussion is in microeconomics context or one may refer to $[2,3]$ for mechanism design review with networks and communications applications. Mechanism design for allocation of a single divisible good in Internet framework has been discussed in $[4,5,6]$, where implementation in general unicast and multicast has been discussed in $[\mathbf{7}, \mathbf{8}, \mathbf{9}, \mathbf{1 0}]$. All of the above as well as the work here uses Nash equilibrium as a solution concept. Nash equilibrium is generally used as a solution concept for complete information games and we discuss this aspect of modelling in section 4 where information assumptions are stated and also in the discussion in section 6.

We consider the problem of multi-rate multicast service provisioning. Here, on the same network, different services with varying QoS within the same service are provided. The main difference with unicast service is that here agents requesting the same service need not be serviced by establishing completely separate connections for each; at common links only connection for the agent with the highest QoS will be established thereby preventing duplication at that link. In fact, multicast formalism subsumes the unicast one for any reasonable system performance metric. Our aim is to build a mechanism who's Nash equilibria give only such allocation that maximises the utilitarian social welfare i.e. sum of agents' utilities. Because of the framework of multicast, there are two different aspects of resource allocation that we come across in this paper - private and public goods. Overall due to the capacity constraints of the links on the network, allocation of rate to one group of users will mean that such additional rate can no longer be allocated to another group of users sharing this link - this is the private good aspect. On the other hand within a group since the allocation via the capacity constraint is dictated only by the highest QoS user from that group on that link, others in the group can be allocated additional rate without having to affect somebody else's allocation - this is the public good aspect.

The philosophy here is to allocate rates based on utilitarian social welfare maximisation, which is a well-known criterion than encompasses fairly general requirements like Pareto optimality and zero independence. In models without strategic users, researchers have argued for different types of maximization criteria. One example is the max-min fairness for multicast is used in $[\mathbf{1 1}, \mathbf{1 2}$, 
13, 14]. On the other hand, in $[15,16]$ authors have used integer and convex programming to get decentralised algorithms that maximise utilitarian social welfare in the multicast problem. One can argue in favour of maximising sum of utilities as follows; any allocation which is paretodominated by another cannot be predicted as the outcome for a society since mere exchange of resources will make everybody better off (a side market would facilitate this and a designer would want to avoid this situation). For quasi-linear utilities e.g. utilities with a linear money component, it can be shown that allocations that maximise sum of utilities (without money) are the only Pareto optimal allocations.

The work here generalises the idea of proportional allocation that was introduced in mechanism design framework by $[\mathbf{5}, \mathbf{6}]$ for the case of infinitely divisible single good (unicast) with only one capacity constraint and for stochastic control of networks by [17]. We emphasize, that in the context of this paper, proportional allocation does not reflect an attempt to allocate resources in a fair manner. It is used as a way to translate users' demands into actual allocations taking into account the capacity constraints. Additionally, readers may be refer to [18] for a full implementation mechanism that uses proportional allocation in the unicast framework. The main advantage of proportional allocation is the off-equilibrium feasibility of allocation. This means that even if agents do not play an equilibrium action profile, the allocation of rates would still satisfy capacity constraints of all links in the network. So the communication system will perform reliably even off-equilibrium; actually the allocation here is always on the boundary of the feasible region thereby always utilising the system resources to the full extent. This is in contrast to [9], where significant effort has been made to ensure budget balance off-equilibrium but feasibility isn't ensured off-equilibrium. The second contribution of this work is to demonstrate how the budget balance property can be added to a non-budget balanced mechanism without significant difficulty (at least in this setup). In section 5 we go on show that with the proportional allocation idea it takes the exchange of one more signal to achieve this. Contrary to [5, 6], the work here ensures full implementation of social welfare maximising allocation, so the designer can guarantee that only the most efficient outcome will be reached and no other (this kind of guarantee is substantially harder to make in a game-theoretic framework).

From a practical point of view, the work here establishes a tight upper bound on the number of message exchanges required for implementation for the multicast problem. The agents here are only required to communicate via announcing signals which consist of demands and prices as opposed to generalised VCG mechanisms (refer to $[\mathbf{1}, \mathbf{2}, \mathbf{1 9}, \mathbf{2 0}]$ or section 5.3 in [21]), which are widely used in mechanism design problems and require announcement of pay-off types (entire valuation function in this case).

The remainder of this paper is structured as follows - in section 2 we state and characterise the solution of the Centralised problem that we wish to implement in a decentralised manner. In sections 4 and 5 we describe and prove our mechanisms for the weak and strong budget 
balance cases respectively. In section 6 we discuss relevant literature and salient features of our mechanism in greater detail.

\section{Centralised Problem}

Formally, maximising social welfare will be defined via the centralised problem below.

Consider a set $\mathcal{N}$ of Internet agents who have been divided into disjoint groups (an agent is considered as a pair of source and destination users). The set of groups is denoted by $\mathcal{K}=$ $\{1,2, \ldots, K\}$ and within a group $k \in \mathcal{K}$, the set of agents by $\mathcal{G}_{k}$. Each group $k \in \mathcal{K}$ has $G_{k}$ agents i.e. $\left|\mathcal{G}_{k}\right|=G_{k}$. From all this one can write $\mathcal{N}=\left\{(k, i) \mid k \in \mathcal{K}, i \in \mathcal{G}_{k}\right\}$ and that the total number of distinct agents is $N=\sum_{k=1}^{K} G_{k}$. The agents communicate over pre-specified routes on the Internet and the agents have been divided into groups based on the content they demand. While the content demanded by different groups is distinct, within a group all agents demand the same content but maybe at different rates. An allocation will be a vector $x$ of rates which has $K=|\mathcal{K}|$ elements, each of which are themselves vectors of sizes $G_{k}, k \in \mathcal{K}$. For this denote by $x_{k i} \in \mathbb{R}_{+}$(where $\mathbb{R}_{+}$is the set of non-negative real numbers) the rate allocated to agent $i$ of group $k$, of the content demanded by group $k$ (from here on we will refer to such an agent as agent $k i$ ). Agent's valuation for an allocation $x$ can be written as

$$
\tilde{v}_{k i}(x)=v_{k i}\left(x_{k i}\right) \quad \forall k i \in \mathcal{N}
$$

where $v_{k i}(\cdot): \mathbb{R}_{+} \rightarrow \mathbb{R}$, for all $k i \in \mathcal{N}$, which indicates that agent $k i$ 's satisfaction depends only on his information rate allocation $x_{k i}$. Due to capacity constraints on the utilised links, allocation to agents is constrained by a number of inequality constraints - both on the network level as well as the group level.

Separate Routes and Notation. Each agent has a fixed pre-determined route. The route $\mathcal{L}_{k i}$ of agent $k i$ is the set of links that agent $k i$ uses for his communication, and $\mathcal{L}=\cup_{k i \in \mathcal{N}} \mathcal{L}_{k i}$ is the set of all available links. The set of agents utilising a link $l \in \mathcal{L}$ is defined as $\mathcal{N}^{l}=\{k i \in$ $\left.\mathcal{N} \mid l \in \mathcal{L}_{k i}\right\}$. Also we define $\mathcal{G}_{k}^{l}=\mathcal{N}^{l} \cap \mathcal{G}_{k}$, the set of agents from group $k$ who use link $l$ and $\mathcal{K}^{l}$ is the set of groups that have at least one agent that uses link $l$ i.e. $\mathcal{K}^{l}=\left\{k \in \mathcal{K} \mid \mathcal{G}_{k}^{l} \neq \emptyset\right\}$. The magnitudes of the sets defined above are $L=|\mathcal{L}|, L_{k i}=\left|\mathcal{L}_{k i}\right|, G_{k}^{l}=\left|\mathcal{G}_{k}^{l}\right|$ and $N^{l}=\left|\mathcal{N}^{l}\right|$.

In addition to group-wise ordering of agents, we also have ordering of agents within the group for every link that is used by that agent. Any agent $k i$, on link $l$, will alternatively be also referred to as

$$
k i \mapsto g_{k}^{l}(i) \quad \text { where } \quad 1 \leq g_{k}^{l}(i) \leq G_{k}^{l}, \quad \forall l \in \mathcal{L}_{k i} .
$$

Here the mapping is such that if for $i, j \in \mathcal{G}_{k}^{l}$ and $i>j$ then $g_{k}^{l}(i)>g_{k}^{l}(j)$. This is done to order agents in a group separately at every link. Note that given the previous definitions, this 
notation is redundant; however we define it because it will be useful later on. Inverse mapping from ordering within a group and link will be denoted by $\left(g_{k}^{l}\right)^{-1}$.

The network administrator is interested in maximizing the social welfare under the link capacity constraints. This centralized problem is formally defined below.

$$
\begin{array}{ll} 
& \max _{x} \sum_{k \in \mathcal{K}} \sum_{i \in \mathcal{G}_{k}} v_{k i}\left(x_{k i}\right) \\
\text { s.t. } & x_{k i} \geq 0 \quad \forall k i \in \mathcal{N} \\
\text { and } & \sum_{k \in \mathcal{K}^{l}} \max _{j \in \mathcal{G}_{k}^{l}}\left\{\alpha_{k j}^{l} x_{k j}\right\} \leq c^{l} \quad \forall l \in \mathcal{L}
\end{array}
$$

Specifically, constraints $C_{2}$ are the inequality constraints on allocation, which as mentioned above, can be interpreted as capacity constraint for every link $l \in \mathcal{L}$, in the network. In this interpretation $\alpha_{k j}^{l}$ would be representative of the QoS requirement of agent $j$ combined with the specific architecture on link $l$. As an example, $\alpha_{k j}^{l}=\frac{1}{R_{k j}\left(1-\epsilon_{k j}^{l}\right)}$ for all links $l \in \mathcal{L}_{k j}$, where $\epsilon_{k j}^{l}$ represents the packet error probability for link $l$ for a packet encoded with channel coding rate $R_{k j}$.

2.1. Assumptions. Our analysis would be done under the following assumptions.

(A1) For all agents, $v_{k i}(\cdot) \in \mathcal{V}_{k i}$, where the sets $\mathcal{V}_{k i}$ are arbitrary subsets of $\mathcal{V}_{0}$, the set of all strictly increasing, strictly concave, twice differentiable functions $\mathbb{R}_{+} \rightarrow \mathbb{R}$ with continuous second derivative.

(A2) $v_{k i}^{\prime}(0)$ is finite $\forall k i \in \mathcal{N}$. This also implies that $v_{k i}^{\prime}(x)$ is finite and bounded $\forall k i$ and $\forall x$ since $v_{k i}$ 's are concave.

(A3) Every link has at least two groups that use it, i.e. $K^{l} \geq 2 \forall l \in \mathcal{L}$.

(A4) The optimal solution of the centralised problem is such that on every link there are at least 2 groups such that each has at least one non-zero component, i.e. if $S^{l}(x):=$ $\left\{k \in \mathcal{K}^{l} \mid \exists i \in \mathcal{G}_{k}^{l}\right.$ s.t. $\left.x_{k i}>0\right\}$ then the assumption says $\left|S^{l}\left(x^{\star}\right)\right| \geq 2 \forall l \in \mathcal{L}$ (where $x^{\star}$ is the optimal solution of $(\mathrm{CP})$ ).

In addition, the coefficients are all strictly positive, i.e. $\alpha_{k i}^{l}>0 \forall l \in \mathcal{L}_{k i}, \forall k i \in \mathcal{N}$. Also, for well-posedness of the problem we take $c^{l}>0 \forall l \in \mathcal{L}$.

Assumption ( $\mathrm{A} 1$ ) is made in order for the centralized problem to have a unique solution and for this solution to be sufficiently characterized by the KKT conditions. (A2) is a mild technical assumption that is required in the proof of Lemma 4.7. Assumption (A3) is made in order to avoid situations where there is a link constraint involving only one agent. Such case requires special handling in the design of the mechanism (since in such a case there is no contention at the link), and destructs from the basic idea that we want to communicate. Finally (A4) is related 
to (A3) and is made in order to simplify the exposition of the proposed mechanism, without having to define corner cases that are of minor importance.

2.2. Necessary and Sufficient Optimality conditions. Following are the KKT conditions, which are generally necessary, but in our case (due to all constraints being affine and strict concavity of $v_{k i}$ ) they will also be sufficient. For this we first rewrite the centralised problem by restating the capacity constraints differently

$$
\begin{array}{ll} 
& \max _{x, m} \sum_{k \in \mathcal{K}} \sum_{i \in \mathcal{G}_{k}} v_{k i}\left(x_{k i}\right) \\
\text { s.t. } & x_{k i} \geq 0 \quad \forall k i \in \mathcal{N} \\
\text { and } \quad & \sum_{k \in \mathcal{K}^{l}} m_{k}^{l} \leq c^{l} \quad \forall l \in \mathcal{L} \\
\text { and } \quad \alpha_{k i}^{l} x_{k i} \leq m_{k}^{l} \quad \forall i \in \mathcal{G}_{k}^{l}, k \in \mathcal{K}^{l}, l \in \mathcal{L}
\end{array}
$$

Here the capacity constraints have been rewritten with the introduction of new variables. The virtual variables $m_{k}^{l}$ represent the weighted maximum rate of group $k$ on link $l$. It's easy to see that the solution of this problem is the same as the solution of the original centralised problem as far as optimal $x$ is concerned. Now we define the Lagrangian for (CP)

$$
\begin{gathered}
L(x, \lambda, \mu, \nu)=\sum_{k \in \mathcal{K}} \sum_{i \in \mathcal{G}_{k}} v_{k i}\left(x_{k i}\right)-\sum_{l \in \mathcal{L}} \lambda_{l}\left(\sum_{k \in \mathcal{K}^{l}} m_{k}^{l}-c^{l}\right) \\
-\sum_{l \in \mathcal{L}} \sum_{k \in \mathcal{K}^{l}} \sum_{i \in \mathcal{G}_{k}^{l}} \mu_{k i}^{l}\left(\alpha_{k i}^{l} x_{k i}-m_{k}^{l}\right)+\sum_{k i \in \mathcal{N}} \nu_{k i} x_{k i}
\end{gathered}
$$

Here KKT conditions will be written without explicitly referring to $\nu_{k i}$ 's and just using the fact that $\nu_{k i}^{\star} \geq 0$ and $\nu_{k i}^{\star} x_{k i}^{\star}=0 \forall k i \in \mathcal{N}$. With the assumptions above, it's easy to see that the KKT conditions below will give rise to a unique $x^{\star}$ (and $m^{\star}$ ) as the optimiser for (CP).

\section{KKT conditions:}

a) Primal Feasibility:

$$
\begin{gathered}
x_{k i}^{\star} \geq 0 \quad \forall k i \in \mathcal{N} \quad \text { and } \quad \sum_{k \in \mathcal{K}^{l}} m_{k}^{l^{\star}} \leq c^{l} \quad \forall l \in \mathcal{L} \\
\text { and } \quad \alpha_{k i}^{l} x_{k i}^{\star} \leq m_{k}^{l \star} \quad \forall i \in \mathcal{G}_{k}^{l}, \quad k \in \mathcal{K}^{l}, l \in \mathcal{L}
\end{gathered}
$$

b) Dual Feasibility: $\lambda_{l}^{\star} \geq 0 \quad \forall l \in \mathcal{L}, \quad \mu_{k i}^{l} \geq 0 \quad \forall k i \in \mathcal{N}^{l}, \quad l \in \mathcal{L}$

c) Complimentary Slackness:

$$
\begin{aligned}
& \lambda_{l}^{\star}\left(\sum_{k \in \mathcal{K}^{l}} m_{k}^{l^{\star}}-c^{l}\right)=0 \quad \forall l \in \mathcal{L} \\
& \mu_{k i}^{l \star}\left(\alpha_{k i}^{l} x_{k i}^{\star}-m_{k}^{l \star}\right)=0 \quad \forall i \in \mathcal{G}_{k}^{l}, k \in \mathcal{K}^{l}, l \in \mathcal{L}
\end{aligned}
$$


d) Stationarity:

$$
\begin{aligned}
& v_{k i}^{\prime}\left(x_{k i}^{\star}\right)=\sum_{l \in \mathcal{L}_{k i}} \mu_{k i}^{l \star} \alpha_{k i}^{l} \quad \forall k i \in \mathcal{N} \quad \text { if } \quad x_{k i}^{\star}>0 \\
& v_{k i}^{\prime}\left(x_{k i}^{\star}\right) \leq \sum_{l \in \mathcal{L}_{k i}} \mu_{k i}^{l \star} \alpha_{k i}^{l} \quad \forall k i \in \mathcal{N} \quad \text { if } \quad x_{k i}^{\star}=0
\end{aligned}
$$

and

$$
\lambda_{l}^{\star}=\sum_{i \in \mathcal{G}_{k}^{l}} \mu_{k i}^{l}{ }^{\star} \quad \forall k \in \mathcal{K}^{l}, l \in \mathcal{L}
$$

Looking at (5), $\mu_{k i}^{l}{ }^{\star}$ will be non-zero only if $\alpha_{k i}^{l} x_{k i}^{\star}=m_{k}^{l \star}$, so these can be interpreted as the "prices" for only those agents who receive maximum weighted allocation from a group at a given link. Consequently, from (6), $\lambda_{l}^{\star}$ will be the sum of $\mu_{k i}^{l}{ }^{\star}$ over those agents in a group for whom it is non-zero and it is the same for all groups. $\lambda_{l}^{\star}$ can be thought of as the common total price subject to each group at link $l$.

\section{Different Formulations of the Centralised Problem}

The designer's task is to ensure that the above optimum allocation is made. This clearly requires the knowledge of $v_{k i}$ 's even when constraints $C_{1}, C_{2}$ and $C_{3}$ are completely known. The premise of our problem is that we are dealing with agents who are strategic and for each of whom, the designer doesn't know their private information i.e. their valuation function $v_{k i}(\cdot)$. One way forward for the designer could be to simply ask each agent to report their private information and announce the solution of (CP), with reported functions in place of $v_{k i}$, for allocation. Apart from the fact that asking to report a function creates a practical communication problem, the main problem with this is that the agents could report untruthfully and end up getting a strictly better allocation. For example, reporting a $v_{k i}$ which has higher derivative than original at every point. In mechanism design terminology, as stated, the allocation function arising out of (CP) isn't even partially implementable ${ }^{1}$. Restricting ourselves to a certain class of utility functions (quasi-linear utilities), provides additional flexibility of penalising agents for reporting untruthfully by imposing taxes/subsidies. In this way, another related problem is created which is implementable, and which we will also show to be equivalent to $(\mathrm{CP})$ as far as allocation is concerned. This leads us to the following additional assumption about agents' utilities

(A5) All agents have quasi-linear utilities, i.e. we can write overall utility functions as

$$
u_{k i}(x, t)=v_{k i}\left(x_{k i}\right)-t_{k i} \quad \forall k i \in \mathcal{N}
$$

where in addition to allocation we have introduced taxes $t$ (a vector like $x$ ).

\footnotetext{
${ }^{1}$ This can be deduced from the revelation principle. Indeed if there was a mechanism that even partially implements the allocation function arising out of (CP), then there would exist also a truthful implementation. However, as shown with the above example, such an implementation will always fail.
} 
Note that under assumption (A5), agent $k i$ pays tax if $t_{k i}>0$ and receives a subsidy if $t_{k i}<0$. Taxes affect utilities linearly and overall utility itself is valuation after adjustment for taxes (total monetary representation of one's state of happiness).

Because we talk about social welfare as our main objective, the centralised problem (CP) isn't complete until we fix who owns the good that is being allocated. Then one will have to further check whether including their welfare in the objective function changes the optimum allocation. As it turns out, under the assumption of quasi-linear utilities and cost of providing the good being zero for the owner, optimum doesn't change even if we involve the seller's welfare. In this regard, there are two interesting ways of reformulating (CP), as elaborated below.

3.1. First Reformulation of CP: Weak budget balance. We now introduce agent 0 as the owner of the good (called the seller). The seller doesn't have any costs for producing and providing the good, i.e. his valuation is the zero function. This could be interpreted as the good being already produced and ready to be provided, so those costs don't come into consideration for the seller as well as the designer. His utility is linear (since valuation is zero) and his revenue is the total tax paid by the agents, $\sum_{k i \in \mathcal{N}} t_{k i}$.

We define centralised problem $\left(\mathrm{CP}_{1}\right)$ as

$$
\begin{aligned}
& \max _{x, m, t} \sum_{k i \in \mathcal{N}} u_{k i}(x, t)+\sum_{k i \in \mathcal{N}} t_{k i} \\
& \text { s.t. } \quad C_{1} \text { and } C_{2} \text { and } C_{3}
\end{aligned}
$$

where now, instead of just taking agent's valuations into account, we maximise the sum of their overall utilities, with the addition of seller's utility (which is only his revenue) - each agent pays a tax $t_{k i}$, all of which goes to the seller, who has no valuation and therefore has utility equal to sum of taxes. Anticipating that a rational seller will only sell if his revenue is non-negative we can add a weak budget balance (WBB) constraint, which states

$$
\sum_{k i \in \mathcal{N}} t_{k i} \geq 0
$$

3.2. Second Reformulation of CP: Strong budget balance. In this case, in contrast to $\left(\mathrm{CP}_{1}\right)$, there is no separate seller. We can alternatively say that the agents are themselves the owners of the good and are only looking to distribute the good (which they collectively own) in a way such that sum of utilities is maximised. Therefore strong budget balance (SBB) constraint is needed. This means that for the system $\mathcal{N}$, no money has been introduced from the outside and the agents wish that no excess money remain on the table either. 
The new centralised problem $\left(\mathrm{CP}_{2}\right)$ resulting from the above interpretation can be stated as $\left(\mathrm{CP}_{2}\right)$

$$
\max _{x, m, t} \sum_{k i \in \mathcal{N}} u_{k i}(x, t)
$$

$$
\begin{array}{ll}
\text { s.t. } C_{1} \text { and } C_{2} \text { and } C_{3} \\
\text { and } \sum_{k i \in \mathcal{N}} t_{k i}=0 .
\end{array}
$$

The two problems defined above will be shown to be equivalent to (CP) where since our original problem (CP) did not involve taxes, we will talk of equivalence only in terms of optimum allocation, $x^{\star}$. Note that due to different conditions on taxes in the two, two different mechanisms will be needed to implement them.

It is straightforward to see that $\left(\mathrm{CP}_{2}\right)$ and $(\mathrm{CP})$ are completely equivalent - due to constraint (SBB), the objective for $\left(\mathrm{CP}_{2}\right)$ is independent of $t$ and is exactly the same as objective for (CP), with same remaining constraints. Now for $\left(\mathrm{CP}_{1}\right)$ and $\left(\mathrm{CP}_{2}\right)$, since the constraints on $x$ are the same in $\left(\mathrm{CP}_{1}\right)$ and $\left(\mathrm{CP}_{2}\right)$ and the $x$-dependent part of the objective in $\left(\mathrm{CP}_{2}\right)$ in independent of $t$ and is the same as the objective of $\left(\mathrm{CP}_{1}\right)$, we can see that $\left(\mathrm{CP}_{1}\right)$ and $\left(\mathrm{CP}_{2}\right)$ are equivalent. The two equivalences above automatically give the third one i.e. $(\mathrm{CP})$ and $\left(\mathrm{CP}_{1}\right)$.

The above equivalences mean that not only will $x^{\star}$ be the same, but also that the necessary and sufficient conditions describing it will be the same i.e. KKT conditions, for $x^{\star}$ and $\lambda^{\star}, \mu^{\star}$, will be exactly the same for all three problems (additionally we will show (WBB) and (SBB) constraints to be satisfied in respective formulations). This fact will be used in Sections 4, 5 where the KKT conditions from Section 2 will be treated as if they have been written for $\left(\mathrm{CP}_{1}\right)$, $\left(\mathrm{CP}_{2}\right)$, respectively.

In Section 4, we will present a mechanism that fully implements $\left(\mathrm{CP}_{1}\right)$ in Nash Equilibria (NE), while in Section 5 we will modify our mechanism to fully implement $\left(\mathrm{CP}_{2}\right)$ in $\mathrm{NE}$.

\section{A Mechanism with Weak Budget Balance}

In this section we refer to $\left(\mathrm{CP}_{1}\right)$ as the centralised problem. So we have all the agents in $\mathcal{N}$ plus the seller and social welfare is in terms of everyone's utility (including seller's).

We will define a mechanism, in a way that doesn't require knowledge of $v_{k i}$, whose game-form will have NE in pure strategies such that the allocation which corresponds to the equilibria of the game-form is same across all equilibria and is equal to the unique optimiser of $\left(\mathrm{CP}_{1}\right), x^{\star}$. In addition, the mechanism will be such that everyone involved (including the seller) will be weakly better-off at equilibrium than not participating at all. 
4.1. Information assumptions. Assume that $v_{k i}(\cdot)$ is a private information of agent $k i$ and nobody else knows it ${ }^{2}$. Let $\mathcal{I}_{c}$ be the set of common information between all agents, containing the information about full rationality of each agent. Finally, let $\mathcal{I}_{d}$ be the knowledge of the designer, containing the information about constraints $C_{1}, C_{2}, C_{3}$, the fact that $\mathcal{V}_{k i} \subset \mathcal{V}_{0}, \forall k i \in \mathcal{N}$ and that the seller has 0 valuation.

4.2. Mechanism. Formally, we have a set of environments $\mathcal{V}=\times_{k i \in \mathcal{N}} \mathcal{V}_{k i}$. We have seen from $\mathrm{KKT}$, how each element of $\mathcal{V}$ can be mapped to an allocation $x^{\star}$ which maximises social welfare for that set of utilities. The allocation $x^{\star}$ achieves the maximum of $\left(\mathrm{CP}_{1}\right)$, and correspondingly any tax $t$ satisfying (WBB) would do.

In our mechanism, the designer would define an action space $\mathcal{S}_{k i}$ for each agent $k i \in \mathcal{N}$. We denote $\mathcal{S}=\times_{k i \in \mathcal{N}} \mathcal{S}_{k i}$ the set of action profiles for all agents. In addition the designer defines and announces the contract $h: S \rightarrow \mathbb{R}_{+}^{N} \times \mathbb{R}^{N}$ that maps every vector of messages received from the agents into an allocation vector and a tax vector (thinking of $x, t$ as vectors with $N=\sum_{k \in \mathcal{K}} G_{k}$ elements). The designer would then ask every agent $k i \in \mathcal{N}$ to choose a message from the set $\mathcal{S}_{k i}$ based on which allocations (and taxes) would be made. The seller is not asked to take any action, so as far as strategic decision making is concerned, we don't need to consider him any further. It is implicit in our mechanism in this section that when the tax $t$ is imposed, the seller gets revenue (or utility) of $\sum_{k i \in \mathcal{N}} t_{k i}$.

Specifically, the designer would ask each agent to report $s_{k i}=\left(y_{k i}, p_{k i}\right)$ where $p_{k i}=$ $\left(\left(p_{k i}^{l}, q_{k i}^{l}\right)\right)_{l \in \mathcal{L}_{k i}}$. This includes their demand for the good and the "price" for each constraint that they are involved, which they believe other(s) should pay. In this for every agent and link, there are two quoted prices - $p_{k i}^{l}$ and $q_{k i}^{l}$; the first one represents the price for $\alpha_{k i}^{l} x_{k i} \leq m_{k}^{l}$ constraint and the second one for the constraint $\alpha_{k j}^{l} x_{k j} \leq m_{k}^{l}$ where $k j$ is the agent that can alternatively be identified by $g_{k}^{l}(i)+1$. All this gives us $\mathcal{S}_{k i}=\mathbb{R}_{+} \times \mathbb{R}_{+}^{2 L_{k i}}$. For received messages $s=\left(s_{11}, \ldots, s_{1 N_{1}}, \ldots, s_{K 1}, \ldots, s_{K N_{K}}\right)=(y, P, Q)=\left(y_{11}, \ldots, y_{K N_{K}}, p_{11} \ldots, p_{K N_{K}}\right)$ the contract $h_{k i}(s)=\left(h_{x, k i}(s), h_{t, k i}(s)\right)$ will be defined for each $k i \in \mathcal{N}$ as follows.

If the received demand vector is $y=\left(y_{11}, \ldots, y_{K N_{K}}\right)=0$ then the allocation is $x=$ $\left(x_{11}, \ldots, x_{K N_{K}}\right)=0$. Otherwise it is evaluated by first generating a scaling factor $r$ through

$$
\begin{gathered}
n_{k}^{l}:=\max _{i \in \mathcal{G}_{k}^{l}}\left\{\alpha_{k i}^{l} y_{k i}\right\} \quad \forall k \in \mathcal{K}^{l}, l \in \mathcal{L} \\
r=\min _{l \in \mathcal{L}} r^{l}
\end{gathered}
$$

\footnotetext{
${ }^{2}$ This assumption is crucial because it raises the question of the validity of NE as a solution concept of the resulting game, since that would require that all agents have complete information about everyone's utilities. We believe this is a serious problem in this entire line of research and that a Bayesian formulation would be more appropriate. However, in this work we accept the justification-weak in our opinion-given by Reichelstein and Reiter in [22] and Groves and Ledyard in [23].
} 


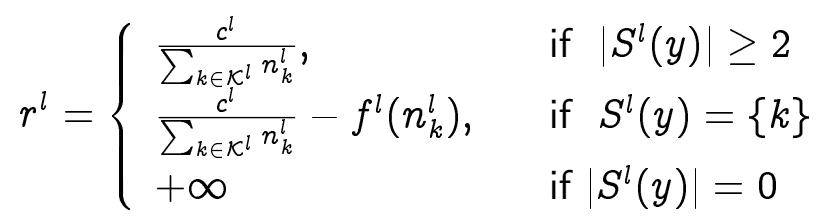

with

$$
f^{l}\left(n_{k}^{l}\right)=f^{l}\left(\left(y_{i}\right)_{i \in \mathcal{G}_{k}^{l}}\right)=\frac{c^{l}}{n_{k}^{l}\left(n_{k}^{l}+1\right)}
$$

Using all these previously defined quantities, the allocation and taxes would be

$$
\begin{gathered}
h_{x, k i}(s)=x_{k i}=r y_{k i} \\
m_{k}^{l}:=r n_{k}^{l} \quad \forall k \in \mathcal{K}^{l}, l \in \mathcal{L}
\end{gathered}
$$

For tax we will first define total prices, $w_{k}^{l}, w_{-k}^{l}$ for any link $l$ and group $k \in \mathcal{K}^{l}$

$$
w_{k}^{l}:=\sum_{i \in \mathcal{G}_{k}^{l}} p_{k i}^{l} \quad \bar{w}_{-k}^{l}:=\frac{1}{\left|\mathcal{K}^{l} \backslash\{k\}\right|} \sum_{k^{\prime} \in \mathcal{K}^{l} \backslash\{k\}} w_{k^{\prime}}^{l}=\frac{1}{K^{l}-1} \sum_{k^{\prime} \in \mathcal{K}^{l} \backslash\{k\}} w_{k^{\prime}}^{l} .
$$

where $\bar{w}_{-k}^{l}$ is well-defined due to assumption (A3).

$$
h_{t, k i}(s)=t_{k i}=\sum_{l \in \mathcal{L}_{k i}} t_{k i}^{l}
$$

where if $G_{k}^{l} \geq 2$ then consider agents $k j$ and $k e$ who have alternate representation on link $l$ as $g_{k}^{l}(i)-1$ and $g_{k}^{l}(i)+1\left(\bmod G_{k}^{l}\right)$, respectively

$$
\begin{gathered}
t_{k i}^{l}=x_{k i} \alpha_{k i}^{l} q_{k j}^{l}+\left(q_{k i}^{l}-p_{k e}^{l}\right)^{2}+\left(w_{k}^{l}-\bar{w}_{-k}^{l}\right)^{2}+q_{k j}^{l}\left(p_{k i}^{l}-q_{k j}^{l}\right)\left(m_{k}^{l}-\alpha_{k i}^{l} x_{k i}\right) \\
+\bar{w}_{-k}^{l}\left(w_{k}^{l}-\bar{w}_{-k}^{l}\right)\left(c^{l}-\sum_{k^{\prime} \in \mathcal{K}^{l}} m_{k^{\prime}}^{l}\right),
\end{gathered}
$$

and if $G_{k}^{l}=1$ then

$$
\begin{gathered}
t_{k i}^{l}=x_{k i} \alpha_{k i}^{l} \bar{w}_{-k}^{l}+\left(w_{k}^{l}-\bar{w}_{-k}^{l}\right)^{2}+\bar{w}_{-k}^{l}\left(p_{k i}^{l}-\bar{w}_{-k}^{l}\right)\left(m_{k}^{l}-\alpha_{k i}^{l} x_{k i}\right) \\
+\bar{w}_{-k}^{l}\left(w_{k}^{l}-\bar{w}_{-k}^{l}\right)\left(c^{l}-\sum_{k^{\prime} \in \mathcal{K}^{l}} m_{k^{\prime}}^{l}\right)
\end{gathered}
$$

Here there are two levels of interactions that the mechanism is dealing with, one among groups for allocation of maximums on each link and second within each group. Here agents are contesting to demand allocation that makes full use of the fact that only maximum at each link will give rise to a price on that link. At any link $l$ and group $k$, total price $w_{k}^{l}$ is the summation of prices quoted by all the agents in the group at link $l$. The quantity $\bar{w}_{-k}^{l}$ is calculated by averaging the total prices for link $l$ over all other groups than $k$. $\left(v_{k i}\right)$, quoting of prices and demand is used as a way of eliciting $v_{k i}^{\prime}\left(x_{k i}\right)$ by comparing it appropriately with prices. In this vein, we do not wish to influence $\bar{p}_{-k}^{l}$ with prices quoted by groups whose agents aren't using the link at all, since the price then essentially doesn't contain any information. 
The quantity $h_{x, k i}(s)$ creates allocation by first creating proxies $n_{k}^{l}$ for weighted maximum at each link for each group. Then $m_{k}^{l}$ 's are created by dilating/shrinking $n_{k}^{l}$ 's on to one of the hyperplanes defined by the second set of constraints in $C_{2}$, specifically, that hyperplane for which the corresponding $m_{k}^{l}$ 's are the closest to origin (this could also be at the intersection of multiple hyperplanes). Finally allocation $x_{k i}$ is calculated by dilating/shrinking $y_{k i}$ by the same factor. Another way to describe this is to say that the contract dilates/shrinks $n_{k}^{l}$ to the boundary of the feasible region defined by the capacity constraints and then allocations within a group are made proportionally. Since all the $\alpha_{k j}^{l}$ 's are positive, this means that all constraints in $C_{2}$ are satisfied for the allocation automatically (shown later). Additionally, the separate definition for $r^{l}$ when $\left|S^{l}(y)\right|<2$ is to ensure (as it will be shown later) that there are no equilibria where $\left|S^{l}(y)\right|<2$. This is required since we are only dealing with achieving solutions ${ }^{3}$ to (CP) which satisfy (A4).

The mechanism gives rise to a one-shot game $\mathfrak{G}$, played by all the agents in $\mathcal{N}$, where action sets are $\left(\mathcal{S}_{k i}\right)_{k i \in \mathcal{N}}$ and utilities are given by

$$
\hat{u}_{k i}(s)=v_{k i}\left(x_{k i}\right)-t_{k i}=v_{k i}\left(h_{x, k i}(s)\right)-h_{t, k i}(s) \quad \forall k i \in \mathcal{N}
$$

We will say that maximising social welfare for $\left(\mathrm{CP}_{1}\right)$ has been fully implemented in $N E$, if the outcomes (all possible NE) of this game produce allocation $x^{\star}$ and all agents in $\mathcal{N}$ plus the seller are better-off participating in the mechanism than opting out (getting 0 allocation and taxes). The second property is known as individual rationality.

\subsection{Results.}

TheOREM 4.1 (Full Implementation). For game $\mathfrak{G}$, there is a unique allocation, $x$, corresponding to all NE. Moreover, $x=x^{\star}$, the maximiser of (CP). In addition, individual rationality is satisfied for all agents and for the seller.

The theorem will be proved by a sequence of results, in which all candidate NE of $\mathfrak{G}$ are characterised by necessary conditions until only one family NE candidates is left. We will then show that $\mathfrak{G}$ has NE in pure strategies, and that all of them result in allocation $x=x^{\star}$. Finally, individual rationality will be checked.

Lemma 4.2 (Primal Feasibility). For any action profile $s=(y, P)$ of game $\mathfrak{G}$, constraints $C_{1}$ and $C_{2}$ are satisfied at the corresponding allocation.

Proof. Constraint $C_{1}$ is clearly always satisfied. For $y=0$ we will have $x=0$ and $m=0$, so constraints $C_{2}$ and $C_{3}$ are also clearly satisfied. We will now show $C_{2}$ and $C_{3}$ for any $y \neq 0$ as demand. In that case $r<+\infty$ (since there exists at least one link $q$ with $\left|S^{q}(y)\right| \geq 1$ and thus $\left.r^{q}<+\infty\right)$. Now, for any link $l$, we have the following two cases. If $\left|S^{l}(y)\right|=0$ then the

\footnotetext{
${ }^{3}$ Note that for the given allocation function, $\left|S^{l}(y)\right|=0,1$ is equivalent to $\left|S^{l}(x)\right|=0,1$.
} 
allocation for all agents on that link is zero (along with the corresponding $m_{k}^{l}$ 's), so $C_{2}$ and $C_{3}$ for those links is satisfied. If $\left|S^{l}(y)\right| \geq 1$ we have

$$
\sum_{k \in \mathcal{K}^{l}} m_{k}^{l}=r \sum_{k \in \mathcal{K}^{l}} n_{k}^{l} \leq r^{l} \sum_{k \in \mathcal{K}^{l}} n_{k}^{l} \leq \frac{c^{l}}{\sum_{k \in \mathcal{K}^{l}} n_{k}^{l}} \sum_{k \in \mathcal{K}^{l}} n_{k}^{l}=c^{l}
$$

where the first inequality holds because $r$ is the minimum of all $r_{l}$ 's. The second inequality will be equality if $\left|S^{l}(y)\right| \geq 2$ and will be strict only if $\left|S^{l}(y)\right|=1$ (see second sub-case in (7)). For $C_{2}$, take any agent $k i$ and link $l \in \mathcal{L}_{k i}$

$$
\alpha_{k i}^{l} x_{k i}=r \alpha_{k i}^{l} y_{k i} \leq r n_{k}^{l}=m_{k}^{l}
$$

where the inequality holds because $n_{k}^{l}$ is the maximum over $\alpha_{k i}^{l} y_{k i}$ 's for all $i \in \mathcal{G}_{k}^{l}$.

Feasibility of allocation for action profiles is a direct consequence of using projections of demand $y$ on to the feasible region. Now we will prove that all groups, using a link, quote the same total price $w_{k}^{l}$ for that link at any equilibrium, this is brought about by the $3^{\text {rd }}$ tax term $\sum_{l}\left(w_{k}^{l}-\bar{w}_{-k}^{l}\right)^{2}$. This is a way of threatening agents with higher taxes just for quoting a different price than average, at each link.

Lemma 4.3. At any $N E s=(y, P)$ of $\mathfrak{G}$, for any link $l \in \mathcal{L}$ we have

$$
w_{k}^{l}=w^{l} \quad \forall k \in \mathcal{K}^{l} .
$$

Also, for any group $k$ and link $l$ such that $G_{k}^{l} \geq 2$ if we take any agents $i, e \in \mathcal{G}_{k}^{l}$ where alternate representation for $e$ is $g_{k}^{l}(i)+1$ then at equilibrium we will have $q_{k i}^{l}=p_{k e}^{l}$. (which will denote as $p_{k, e}^{l}$ )

Proof. First we will show the second part of the lemma, so suppose there are agents $i, e \in \mathcal{G}_{k}^{l}$ as above, for whom $q_{k i}^{l} \neq p_{k e}^{l}$. Here if agent $k i$ deviates with $q_{k i}^{l}{ }^{\prime}=p_{k e}^{l}$ then we can write the difference in agent $k i$ 's utility after and before deviation by just comparing tax for link $l$ (since allocation and tax for other links don't change)

$$
\Delta \hat{u}_{k i}=-\left(q_{k i}^{l}{ }^{\prime}-p_{k e}^{l}\right)^{2}+\left(q_{k i}^{l}-p_{k e}^{l}\right)^{2}=\left(q_{k i}^{l}-p_{k e}^{l}\right)^{2}>0
$$

which means that the deviation was profitable. This gives us the second part of the lemma. (In addition to defining $q_{k i}^{l}=p_{k e}^{l}=p_{k, e}^{l}$ when $G_{k}^{l} \geq 2$ we will also denote $p_{k i}^{l}=w_{k}^{l}=p_{k, i}^{l}$ when $\left.\mathcal{G}_{k}^{l}=\{i\}\right)$.

For the first part, suppose there is a link $l$ for which $\left(w_{k}^{l}\right)_{k \in \mathcal{K}^{l}}$ are not all equal, at equilibrium. Clearly then there is a group $k \in \mathcal{K}^{l}$ for which $w_{k}^{l}>\bar{w}_{-k}^{l}$ (this can be seen from (9)). We will show that some agent $i \in \mathcal{G}_{k}^{l}$ can deviate by reducing price $p_{k i}^{l}$ and be strictly better off, thereby contradicting the equilibrium condition. First we will take the case when the group $k$ is such that $G_{k}^{l} \geq 2$ and then $G_{k}^{l}=1$. 
Since $w_{k}^{l}>\bar{w}_{-k}^{l}$ we must have $w_{k}^{l}>0$ and since $w_{k}^{l}=\sum_{i \in \mathcal{G}_{k}^{l}} p_{k i}^{l}$ there must be an agent $i \in \mathcal{G}_{k}^{l}$ for whom $p_{k i}^{l}>0$. Take deviation by this agent $k i$ as $p_{k i}^{l}{ }^{\prime}=p_{k i}^{l}-\epsilon>0$, for which we can write the difference in utility, just as before, as

$$
\begin{aligned}
& \Delta \hat{u}_{k i}=-\left(w_{k}^{l}-\bar{w}_{-k}^{l}-\epsilon\right)^{2}+\left(w_{k}^{l}-\bar{w}_{-k}^{l}\right)^{2}-p_{k, i}^{l}\left(p_{k, i}^{l}-\epsilon-p_{k, i}^{l}\right)\left(m_{k}^{l}-\alpha_{k i}^{l} x_{k i}\right) \\
& +0-\bar{w}_{-k}^{l}\left(w_{k}^{l}-\epsilon-\bar{w}_{-k}^{l}\right)\left(c^{l}-\sum_{k \in \mathcal{K}^{l}} m_{k}^{l}\right)+\bar{w}_{-k}^{l}\left(w_{k}^{l}-\bar{w}_{-k}^{l}\right)\left(c^{l}-\sum_{k \in \mathcal{K}^{l}} m_{k}^{l}\right) \\
& =-\epsilon^{2}+2 \epsilon\left(w_{k}^{l}-\bar{w}_{-k}^{l}\right)+\epsilon p_{k, i}^{l}\left(m_{k}^{l}-\alpha_{k i}^{l} x_{k i}\right)+\epsilon \bar{w}_{-k}^{l}\left(c^{l}-\sum_{k \in \mathcal{K}^{l}} m_{k}^{l}\right) \\
& (11) \Delta \hat{u}_{k i}=\epsilon\left(-\epsilon+2\left(w_{k}^{l}-\bar{w}_{-k}^{l}\right)+p_{k, i}^{l}\left(m_{k}^{l}-\alpha_{k i}^{l} x_{k i}\right)+\bar{w}_{-k}^{l}\left(c^{l}-\sum_{k \in \mathcal{K}^{l}} m_{k}^{l}\right)\right)=\epsilon(-\epsilon+a)
\end{aligned}
$$

where $a>0$ because of Lemma 4.2 and the fact that $w_{k}^{l}>\bar{w}_{-k}^{l}$. So by taking $\epsilon$ such that $\min \left\{a, p_{k i}^{l}\right\}>\epsilon>0$, the above deviation will be a profitable one for agent $k i$. This gives the result for $G_{k}^{l} \geq 2$.

For $G_{k}^{l}=1$, say $\mathcal{G}_{k}^{l}=\{i\}$, we have that $p_{k i}^{l}=w_{k}^{l}>\bar{w}_{-k}^{l}$. This again means that $p_{k i}^{l}>0$ and we take the deviation $p_{k i}^{l}{ }^{\prime}=p_{k i}^{l}-\epsilon>0$ and get

$$
\Delta \hat{u}_{k i}=\epsilon\left(-\epsilon+2\left(w_{k}^{l}-\bar{w}_{-k}^{l}\right)+\bar{w}_{-k}^{l}\left(m_{k}^{l}-\alpha_{k i}^{l} x_{k i}\right)+\bar{w}_{-k}^{l}\left(c^{l}-\sum_{k \in \mathcal{K}^{l}} m_{k}^{l}\right)\right) .
$$

Following the same argument as above we will get our result here as well.

With Lemma 4.3, we can talk in terms of the common total price vector at equilibrium rather than different total price vectors for all agents. In particular, any NE $s=(y, P, Q)$ can be characterized as $s=(y, P)$ with $P=\left(p_{k, i}\right)_{k i \in \mathcal{N}}$ and $p_{k, i}=\left(p_{k, i}^{l}\right)_{l \in \mathcal{L}_{k i}}$.

Later it will become clear how $p_{k, i}^{l}$ and $w^{l}$ take the place of dual variables $\mu_{k i}^{l}$ and $\lambda_{l}$ when we compare equilibrium conditions with KKT conditions, hence we identify the following condition as dual feasibility.

Lemma 4.4 (Dual Feasibility). $p_{k, i}^{l} \geq 0, w^{l} \geq 0 \quad \forall i \in \mathcal{G}_{k}^{l}, \forall k \in \mathcal{K}^{l}$ and $\forall l \in \mathcal{L}$.

ProOF. This is also by design, since agents are only allowed to quote non-negative prices and that $w^{l}$ is the sum of such prices.

Following is the property that solidifies the notion of prices as dual variables, since here we claim that inactive constraints do not contribute to payment at equilibrium. This notion is very similar to the centralised problem, where if we know certain constraints to be inactive at the optimum then the same problem without these constraints would be equivalent to the original. The $4^{\text {th }}$ and $5^{\text {th }}$ terms in the tax function facilitate this by charging extra taxes for inactive 
constraints where the agent is quoting higher prices than the average of remaining ones, thereby driving prices down.

Lemma 4.5 (Complimentary Slackness). At any NE $s=(y, P)$ of game $\mathfrak{G}$ with corresponding allocation $x$, for any agent $i \in \mathcal{G}_{k}^{l}$, group $k \in \mathcal{K}^{l}$ and link $l \in \mathcal{L}$ we have

$$
w^{l}\left(\sum_{k \in \mathcal{K}^{l}} m_{k}^{l}-c^{l}\right)=0, \quad p_{k, i}^{l}\left(\alpha_{k i}^{l} x_{k i}-m_{k}^{l}\right)=0
$$

PROOF. Suppose there is a link $l$ for which $w^{l}>0$ and $\sum_{k \in \mathcal{K}^{l}} m_{k}^{l}<c^{l}$. Take any group $k \in \mathcal{K}^{l}$ and an agent $i \in \mathcal{G}_{k}^{l}$ such that $p_{k i}^{l}=p_{k, i}^{l}>0$ (there is such an agent because $w^{l}=\sum_{i \in \mathcal{G}_{k}{ }^{l}} p_{k i}^{l}>0$ ). Take the deviation $p_{k i}^{l}{ }^{\prime}=p_{k, i}^{l}-\epsilon>0$ and we get (using same arguments as in (11) and noting that $w_{k}^{l}=\bar{w}_{-k}^{l}=w^{l}$ )

$$
\Delta \hat{u}_{k i}=\epsilon(-\epsilon+\underbrace{p_{k, i}^{l}\left(m_{k}^{l}-\alpha_{k i}^{l} x_{k i}\right)}_{\geq 0 \text { by Lemma } 4.2}+w^{l}\left(c^{l}-\sum_{k \in \mathcal{K}^{l}} m_{k}^{l}\right))=\epsilon(-\epsilon+a) .
$$

where $a>0$ due to Lemma 4.2 and the assumption that $w^{l}\left(c^{l}-\sum_{k \in \mathcal{K}^{l}} m_{k}^{l}\right)>0$. This gives us that $w^{l}\left(c^{l}-\sum_{k \in \mathcal{K}^{l}} m_{k}^{l}\right)=0$ for all $l \in \mathcal{L}$ at equilibrium.

Now suppose there is an agent $k i$ for whom $p_{k i}^{l}=p_{k, i}^{l}>0$ and $\alpha_{k i}^{l} x_{k i}<m_{k}^{l}$. Same as before, we will take the deviation $p_{k i}^{l}{ }^{\prime}=p_{k i}^{l}-\epsilon>0$,

$$
\Delta \hat{u}_{k i}=\epsilon\left(-\epsilon+p_{k, i}^{l}\left(m_{k}^{l}-\alpha_{k i}^{l} x_{k i}\right)\right)=\epsilon(-\epsilon+a)
$$

where $a>0$ by assumption. This gives us that $p_{k, i}^{l}\left(m_{k}^{l}-\alpha_{k i}^{l} x_{k i}\right)=0$ for all $k i \in \mathcal{N}^{l}$ and $l \in \mathcal{L}$, at equilibrium.

Lemma 4.6 (Stationarity). At any NE $s=(y, P)$ of game $\mathfrak{G}$, and corresponding allocation, $x$, we have

$$
\begin{aligned}
& v_{k i}^{\prime}\left(x_{k i}\right)=\sum_{l \in \mathcal{L}_{k i}} p_{k, i}^{l} \alpha_{k i}^{l} \quad \forall k i \in \mathcal{N} \quad \text { if } \quad x_{k i}>0 \\
& v_{k i}^{\prime}\left(x_{k i}\right) \leq \sum_{l \in \mathcal{L}_{k i}} p_{k, i}^{l} \alpha_{k i}^{l} \quad \forall k i \in \mathcal{N} \quad \text { if } \quad x_{k i}=0
\end{aligned}
$$

and

$$
w^{l}=\sum_{i \in \mathcal{G}_{k}^{l}} p_{k, i}^{l} \quad \forall k \in \mathcal{K}^{l}, \forall l \in \mathcal{L}
$$

PROOF. (12) is true by construction since we defined $w_{k}^{l}=\sum_{i \in \mathcal{G}_{k}^{l}} p_{k i}^{l}$ and by Lemma 4.3 we have $p_{k, i}^{l}=p_{k i}^{l}$ and $w_{k}^{l}=w^{l}$.

At any NE, agent $k i^{\prime} s$ utility in the game $\hat{u}_{k i}\left(s^{\prime}\right)=v_{k i}\left(h_{x, k i}\left(s^{\prime}\right)\right)-h_{t, k i}\left(s^{\prime}\right)$ as a function of his message $s_{k i}^{\prime}=\left(y_{k i}^{\prime}, p_{k i}^{\prime}\right)$, with $s_{-k i}$ fixed, should have a global maximum at $s_{k i}=\left(y_{k i}, p_{k i}\right)$. This would mean that if this function was differentiable w.r.t. $y_{k i}^{\prime}$ at $s$, the partial derivatives 
w.r.t. $y_{k i}^{\prime}$ at $s$ should be 0 . However, since our allocation dilates/shrinks demand vector $y^{\prime}$ on to the feasible region, it could be the case that increasing and decreasing $y_{k i}^{\prime}$ gives allocations lying on different hyperplanes, meaning that the transformation from $y^{\prime}$ to $x^{\prime}$ is different on both sides of $y_{k i}$ and therefore we conclude that $\hat{u}_{k i}$ could be non-differentiable w.r.t $y_{k i}^{\prime}$ at $s$. Important thing here however is to notice that right and left derivatives exist, it's just that they may not be equal. Hence we can take derivatives on both sides of $y_{i}$ as (noting that derivative of the other terms in utility involving $x_{k i}$ or involving $m_{k}^{l}$ will be zero due Lemma 4.3)

$$
\begin{aligned}
& \left.\frac{\partial \hat{u}_{k i}{ }^{\prime}}{\partial y_{k i}}\right|_{y_{k i}^{\prime} \downarrow y_{k i}}=\left.\left(v_{k i}^{\prime}\left(x_{k i}\right)-\sum_{l \in \mathcal{L}_{k i}} p_{k, i}^{l} \alpha_{k i}^{l}\right) \frac{\partial x_{k i}^{\prime}}{\partial y_{k i}^{\prime}}\right|_{y_{k i}^{\prime} \downarrow y_{k i}} \\
& \left.\frac{\partial \hat{u}_{k i}{ }^{\prime}}{\partial y_{k i}}\right|_{y_{k i}^{\prime} \uparrow y_{k i}}=\left.\left(v_{k i}^{\prime}\left(x_{k i}\right)-\sum_{l \in \mathcal{L}_{k i}} p_{k, i}^{l} \alpha_{k i}^{l}\right) \frac{\partial x_{k i}^{\prime}}{\partial y_{k i}^{\prime}}\right|_{y_{k i}^{\prime} \uparrow y_{k i}}
\end{aligned}
$$

We will first show that the $\partial x_{i} / \partial y_{i}$ term above (for either equation) is always positive. If $y=0$ then clearly this is true, because if any agent $k i$ demands $y_{k i}>\epsilon$ while $y_{-k i}=0$ then clearly $x_{k i}>0$ (in fact the allocation is differentiable at $y=0$ ). If $y \neq 0$ from (8), we can write

$$
\beta:=\frac{\partial x_{k i}}{\partial y_{k i}}=\frac{\partial\left(r y_{k i}\right)}{\partial y_{k i}}=r+y_{k i} \frac{\partial r}{\partial y_{k i}}=r^{q}+y_{k i} \frac{\partial r^{q}}{\partial y_{k i}}
$$

where $r=r^{q}$.

From here we divide our arguments into following cases: (A) $k i \notin \mathcal{N}^{q}$ ( $\left.\Leftrightarrow i \notin \mathcal{G}_{k}^{q}\right)$; (B) $k i \in \mathcal{N}^{q}, i \notin \arg \max _{j \in \mathcal{G}_{k}^{q}}\left\{\alpha_{k j}^{q} y_{k j}\right\}$ and $(\mathrm{C}) k i \in \mathcal{N}^{q}, i \in \arg \max _{j \in \mathcal{G}_{k}^{q}}\left\{\alpha_{k j}^{q} y_{k j}\right\}$.

(A) Here clearly $\partial r^{q} / \partial y_{i}=0$ and this makes $\beta=r^{q}>0$.

(B) Since value of $r^{q}$ depends only on the value of $\left(n_{k}^{q}\right)_{k \in \mathcal{K}^{q}}$, and in this case changes in $y_{k i}$ don't affect $n_{k}^{q}$ we can see that $\partial r^{q} / \partial y_{k i}=0$ and so $\beta=r^{q}>0$.

(C) We divide this case into two cases: $\left|S^{q}(y)\right| \geq 2$ or $\left|S^{q}(y)\right|=1$. If $\left|S^{q}(y)\right| \geq 2$ then

$$
\beta=r^{q}+y_{k i} \frac{\partial r^{q}}{\partial y_{k i}}=r^{q}+y_{k i}\left(-\frac{c^{q}}{\left(\sum_{k_{0} \in \mathcal{K}^{q}} n_{k_{0}}^{q}\right)^{2}}\right) \frac{\partial n_{k}^{q}}{\partial y_{k i}} .
$$

Here $\frac{\partial n_{k}^{q}}{\partial y_{k i}}$ is either $\alpha_{k i}^{q}$ or 0 . If it is 0 then $\beta=r^{q}>0$. Otherwise we have

$$
\beta=\frac{\left(r^{q}\right)^{2}}{c^{q}} \sum_{k_{0} \in \mathcal{K}^{q} \backslash\{k\}} n_{k_{0}}^{q}
$$

which is positive because $\left|S^{q}(y)\right| \geq 2$, since then there is at least one positive term in the summation. For $\left|S^{q}(y)\right|=1$, we will consider $S^{q}(y)=\{k\}$; else in case $S^{q}(y)=\left\{k_{0}\right\} \neq\{k\}$, taking the derivative would give the same expression as above. For $S^{q}(y)=\{k\}$ we will get

$$
r^{q}=\frac{c^{q}}{n_{k}^{q}}-\frac{c^{q}}{n_{k}^{q}\left(n_{k}^{q}+1\right)}=\frac{c^{q}}{n_{k}^{q}}\left(1-\frac{1}{n_{k}^{q}+1}\right)=\frac{c^{q}}{n_{k}^{q}+1}
$$




$$
\beta=r^{q}+y_{k i} \frac{\partial r^{q}}{\partial n_{k}^{q}} \frac{\partial n_{k}^{q}}{\partial y_{k i}}
$$

where $\frac{\partial n_{k}^{q}}{\partial y_{k i}}$ is either 0 or $\alpha_{k i}^{q}$. If it is 0 then $\beta=r^{q}>0$ and if it is $\alpha_{k i}^{q}$ then we have

$$
\beta=\frac{\left(r^{q}\right)^{2}}{c^{q}}
$$

So we have $\beta>0$ in all cases.

Referring to (13), there are two possibilities, the first term on RHS in both equations in (13) is positive or negative. If it's positive, then we can see from the first equation in (13) that by increasing $y_{k i}^{\prime}$ from $y_{k i}$ (and therefore $x_{k i}^{\prime}$ from $x_{k i}$ ) agent $k i$ can increase his pay-off, which contradicts equilibrium. Now similarly consider the first term in (13) to be negative, then from the second equation in (13), agent $k i$ can reduce $y_{k i}^{\prime}$ from $y_{k i}$ to get a better pay-off. But the downward deviation in $y_{k i}^{\prime}$ is only possible if $y_{k i}>0\left(\Leftrightarrow x_{k i}>0\right)$. So we conclude that

$$
\begin{aligned}
& v_{k i}^{\prime}\left(x_{k i}\right)=\sum_{l \in \mathcal{L}_{k i}} p_{k, i}^{l} \alpha_{k i}^{l} \quad \forall k i \in \mathcal{N} \quad \text { if } \quad x_{k i}>0 \\
& v_{k i}^{\prime}\left(x_{k i}\right) \leq \sum_{l \in \mathcal{L}_{k i}} p_{k, i}^{l} \alpha_{k i}^{l} \quad \forall k i \in \mathcal{N} \quad \text { if } \quad x_{k i}=0
\end{aligned}
$$

Collecting the results of the above lemmas, we can conclude that every NE satisfies the KKT conditions of the (CP). This means we now have necessary conditions on the NE up to the point of having unique allocation. In the next Lemma we verify the existence of the equilibria that we have claimed.

LEMma 4.7 (Existence). For the game $\mathfrak{G}$, there exists equilibria $s=(y, P, Q)=(y, P)$, where corresponding allocation $\left(x_{k i}\right)_{k i \in \mathcal{N}}$ and prices $\left(p_{k, i}^{l}\right)_{k i \in \mathcal{N}^{l}, l \in \mathcal{L}}$ and $\left(w^{l}\right)_{l \in \mathcal{L}}$ satisfy KKT conditions as $\left(x_{k i}^{\star}\right)_{k i \in \mathcal{N}},\left(\mu_{k i}^{l}{ }^{\star}\right)_{k i \in \mathcal{N}^{l}, l \in \mathcal{L}}$ and $\left(\lambda_{l}^{\star}\right)_{l \in \mathcal{L}}$, respectively.

ProOF. The proof is completed in two parts. Firstly we will check that for every $x$ that can be a possible solution to (CP) while satisfying assumption (A4) there is indeed at least one $y \in \mathbb{R}_{+}^{N}$ such that the allocation corresponding to $y$ is $x$. (However we do not need to check the same for prices and Lagrange multipliers $(\lambda, \mu)$ since there it is straightforward). Secondly we will check that for the claimed NE, there are no unilateral deviations that are profitable.

In lieu of (A4), the optimal $x^{\star}$ is such that $\left|S^{l}\left(x^{\star}\right)\right| \geq 2$ for all links; also it is clear that $x^{\star}$ and $m^{\star}$ are on the boundary of the feasible region defined by $C_{1}$ and $C_{2}$. So any vector $y$ which is a scalar multiple of $x^{\star}$ would give allocation $x^{\star}$ (and corresponding $m$ will be equal to $m^{\star}$ ). So in particular, $y=x$ will also do the job. Hence our first task is done. (kindly see more detailed comments at the end of this proof for why (A4) was required here)

Now we will check for profitable deviations. For this we want to show any action profile that satisfies the hypothesis of above statement, is a NE. Due to assumptions on $v_{i}$ and by 
construction, where we have taxes and allocations that are continuous, so we can do this by checking the first and second order conditions for any arbitrary agent, say agent $k i$. Price derivatives are

$$
\frac{\partial \hat{u}_{k i}}{\partial p_{k i}^{l}}=-2\left(w_{k}^{l}-\bar{w}_{-k}^{l}\right)-q_{k j}^{l}\left(m_{k}^{l}-\alpha_{k i}^{l} x_{k i}\right)-\bar{w}_{-k}^{l}\left(c^{l}-\sum_{k^{\prime} \in \mathcal{K}^{l}} m_{k^{\prime}}^{l}\right) \quad \forall l \in \mathcal{L}_{k i} \text { s.t. } G_{k}^{l} \geq 2
$$

This is clearly zero at equilibrium due to Lemma 4.3 and 4.5. Similarly we can get price derivative equal to 0 when $G_{k}^{l}=1$. Now referring to the differentiability arguments made in the proof of Lemma 4.6, we symbolically write

$$
\begin{gathered}
\frac{\partial \hat{u}_{k i}}{\partial y_{k i}}=\left(v_{k i}^{\prime}\left(x_{k i}\right)-\sum_{l \in \mathcal{L}_{k i}} \alpha_{k i}^{l} q_{k j}^{l}\right)\left(\frac{\partial x_{k i}}{\partial y_{k i}}\right)-\sum_{l \in \mathcal{L}_{k i}} q_{k j}^{l}\left(p_{k i}^{l}-q_{k j}^{l}\right)\left(\frac{\partial m_{k}^{l}}{\partial y_{k i}}-\alpha_{k i}^{l} \frac{\partial x_{k i}}{\partial y_{k i}}\right) \\
-\sum_{l \in \mathcal{L}_{k i}} \bar{w}_{-k}^{l}\left(w_{k}^{l}-\bar{w}_{-k}^{l}\right)\left(-\sum_{k^{\prime} \in \mathcal{K}^{l}} \frac{\partial m_{k^{\prime}}^{l}}{\partial y_{k i}}\right)
\end{gathered}
$$

where as before we note that $\frac{\partial x_{k i}}{\partial y_{k i}}>0$, always (note that we have written the above expression as if all links have $G_{k}^{l} \geq 2$ but the expression and consequent arguments won't change much even if there are links where $G_{k}^{l}=1$ ). The second and third terms above are always zero at equilibrium (due to Lemma 4.3). So if $x_{k i}>0$ at equilibrium then the 1 st term is also zero as well (due to Stationarity) and if $x_{k i}=0\left(\Leftrightarrow y_{k i}=0\right)$ the 1 st term is either negative, making the whole derivative negative (this is fine since downward deviation isn't possible from $y_{k i}=0$ ), or equal to zero. For the remaining of the proof we will consider the case where the first order derivatives are equal to zero.

Second order partial derivatives are

$$
\begin{gathered}
u_{p p}:=\frac{\partial^{2} \hat{u}_{k i}}{\partial p_{k i}^{l} \partial p_{k i}^{l}}=-2 \quad u_{p l 12}:=\frac{\partial^{2} \hat{u}_{k i}}{\partial p_{k i}^{l_{1}} \partial p_{k i}^{l_{2}}}=0 \\
u_{p q}:=\frac{\partial^{2} \hat{u}_{k i}}{\partial p_{k i}^{l} \partial q_{k i}^{l}}=0 \quad u_{p q 12}:=\frac{\partial^{2} \hat{u}_{k i}}{\partial p_{k i}^{l_{1}} \partial q_{k i}^{l_{2}}}=0 \\
u_{q q}:=\frac{\partial^{2} \hat{u}_{k i}}{\partial q_{k i}^{l} \partial q_{k i}^{l}}=-2 \quad u_{q l k}:=\frac{\partial^{2} \hat{u}_{k i}}{\partial q_{k i}^{l} \partial q_{k i}^{k}}=0 \quad u_{q y}:=\frac{\partial^{2} \hat{u}_{k i}}{\partial q_{k i}^{l} \partial y_{k i}}=0 \\
u_{y y}:=\frac{\partial^{2} \hat{u}_{k i}}{\partial y_{k i} \partial y_{k i}}=\left(v_{k i}^{\prime}\left(x_{k i}\right)-\sum_{l \in \mathcal{L}_{k i}} \alpha_{k i}^{l} q_{k j}^{l}\right)\left(\frac{\partial^{2} x_{k i}}{\partial y_{k i}^{2}}\right)+v_{k i}^{\prime \prime}\left(x_{k i}\right)\left(\frac{\partial x_{k i}}{\partial y_{k i}}\right) \\
u_{p y}:=\frac{\partial^{2} \hat{u}_{k i}}{\partial p_{k i}^{l} \partial y_{k i}}=q_{k j}^{l}\left(\alpha_{k i}^{l} \frac{\partial x_{k i}}{\partial y_{k i}}-\frac{\partial m_{k}^{l}}{\partial y_{k i}}\right)+\bar{w}_{-k}^{l}\left(\sum_{k^{\prime} \in \mathcal{K}^{l}} \frac{\partial m_{k^{\prime}}^{l}}{\partial y_{k i}}\right)
\end{gathered}
$$

These derivatives will give us a Hessian $H$ of size $\left(2 L_{k i}+1\right) \times\left(2 L_{k i}+1\right)$, where 1 st row and column represent $y_{k i}$ and subsequent $L_{k i}$ rows and columns represent $p_{k i}^{l}$ 's for different $l$ 's and 
then the last $L_{k i}$ rows represent $q_{k i}^{l}$ 's. We want $H$ to be negative definite at equilibrium. Now, 1 st term in $u_{y y}$ is zero at equilibrium, and the 2 nd term is strictly negative due to strict concavity of $v_{i}$. This along with $u_{p p}=u_{q q}=-2$ tells us that all diagonal entries in $H$ are negative. Also notice that all off-diagonal entries, except the first $L_{k i}+1$ in the first row and column, are zero. Finally, note that due to assumption (A2), all prices are finite at equilibrium and so $u_{p y}$ will be finite. We will show that roots of the characteristic polynomial of $H$ (i.e. its eigenvalues) all become negative $\forall y$ such that $|y|$ sufficiently large.

For this, we take a generic matrix $A_{0}$, which is similar in structure to $H$ and has the same dependence on $|y|$ as $H$. So $A_{0}$ will be of the form

$$
A_{0}=\left[\begin{array}{ll}
A & 0 \\
0 & D
\end{array}\right]
$$

where $D=(-2) I_{L_{k i}}$. In this case we know that eigenvalues of $A$ and $D$ together will give us all the eigenvalues of $A_{0}$. Clearly eigenvalues of $D$ are -2 repeated $L_{k i}$ times, so all that we now need to do is check whether all eigenvalues of $A$ are negative. Entries in $A$ are

$$
\begin{aligned}
& a_{11}=-\frac{a}{|y|} \quad a_{i j}=a_{j i}=0 \quad \forall i, j>1, i \neq j \\
& a_{i i}=-2 \quad a_{1 i}=a_{i 1}=\frac{b_{i-1}}{|y|} \quad \forall \quad 2 \leq i \leq L_{k i}+1
\end{aligned}
$$

where $a>0$ (and we don't care about the sign of $b_{i}{ }^{\prime}$ 's). We can explicitly calculate $|A-\lambda I|$ and write the characteristic equation as

$$
Q(\lambda)=\left(-\frac{a}{|y|}-\lambda\right)(-2-\lambda)^{L_{k i}}+\frac{\sum_{i=1}^{L_{k i}}(-1)^{i} b_{i}^{2}}{|y|^{2}}(-2-\lambda)^{L_{k i}-1}=0
$$

So -2 is a repeated eigenvalue, $L_{k i}-1$ times. The equation for the remaining two roots can be written as

$$
\left(-\frac{a}{|y|}-\lambda\right)(-2-\lambda)+\frac{C}{|y|^{2}}=0
$$

Necessary and sufficient conditions for both roots of this quadratic to be negative are

$$
\left(2+\frac{a}{|y|}\right)>0 \quad \frac{2 a}{|y|}+\frac{C}{|y|^{2}}>0
$$

first of which is always true, since $a>0$. The second one gives $|y|>\frac{-C}{2 a}$, which can be satisfied (for large enough $|y|$ ) irrespective of the sign of constant $C$.

Hence we have shown the Hessian $H$ to be negative definite for $|y|$ large enough.

Several comments are in order regarding the selection of the proportional allocation mechanism and in particular (7). If we use "pure" proportional allocation i.e. same expression for $r^{l}$ for $\left|S^{l}(y)\right| \geq 2$ and $\leq 1$, then irrespective of optimal solution of $(\mathrm{CP})$, for game $\mathfrak{G}$ the "stationarity" 
property will not be satisfied for equilibria with $\left|S^{l}(y)\right| \leq 1$. Thus the mechanism will result in additional extraneous equilibria. For this reason we tweak the expression for $r^{l}$ when $\left|S^{l}(y)\right| \leq 1$, so that we can eliminate these extraneous equilibria - irrespective of the solution of (CP). With this tweak in the expression for $r^{l}$, all KKT conditions become necessary for all equilibria regardless of the value of $\left|S^{l}(y)\right|$. This however creates a problem in the proof of existence of equilibria. In particular, if $x^{\star}$ was such that it had links where $\left|S^{l}\left(x^{\star}\right)\right|=1$ then in our allocation this would require $y$ at NE such that $\left|S^{l}(y)\right|=1$. In this case the $r^{l}$ used would be lower than what the proportional allocation requires (see second sub-case in (7)) and we actually would have the problem of possibly not having any $y$ that creates $x^{\star}$ as allocation. Hence we have used (A4) to eliminate this case.

Lemma 4.8 (Individual Rationality). At any $N E s=(y, P)$ of $\mathfrak{G}$, with corresponding allocation $x$ and taxes $t$, we have

$$
\begin{aligned}
u_{k i}(x, t) & \geq u_{k i}(0,0) \quad \forall k i \in \mathcal{N} \\
\text { and } \quad \sum_{k i \in \mathcal{N}} t_{k i} & \geq 0 \quad(W B B)
\end{aligned}
$$

ProOF. Because of Lemma 4.3, the only non-zero term in $t_{k i}$ (see (10)) at equilibrium is $x_{k i} \sum_{l \in \mathcal{L}_{k i}} \alpha_{k i}^{l} p_{k, i}^{l}$, which is clearly non-negative. Hence $\sum_{k i \in \mathcal{N}} t_{k i} \geq 0$ at equilibrium. This is the seller's individual rationality condition.

Now if $x_{k i}=0$ then we know from Lemma 4.3 and (10) that $t_{k i}=0$ and so (14) is evident. Now take $x_{k i}>0$ and define the function

$$
f(z)=v_{k i}(z)-z \sum_{l \in \mathcal{L}_{k i}} \alpha_{k i}^{l} p_{k, i}^{l}
$$

Note that $f(0)=u_{k i}(0,0)$ and $f\left(x_{k i}\right)=u_{k i}(x, t)$, the utility at equilibrium. Since $f^{\prime}\left(x_{k i}\right)=0$ (Lemma 4.6), we see that $\forall 0<y<x_{k i}, f^{\prime}(y)>0$ since $f$ strictly concave (because of $v_{k i}$ ). This clearly tells us $f\left(x_{k i}\right) \geq f(0)$.

Now that we have Lemmas characterising NE in the same way as KKT conditions (and individual rationality), we can compare them to prove Theorem 4.1.

Proof of TheOREM 4.1. We know that the four KKT conditions produce a unique solution $x^{\star}$ (and corresponding $\lambda^{\star}$ ). For the game $\mathfrak{G}$, from Lemmas 4.2-4.6 we can see that at any $\mathrm{NE}$, allocation $x$ and prices $p$ satisfy the same conditions as the four KKT conditions and hence they give a unique $x=x^{\star}$, as long as (A5) is satisfied. So we have that the allocation is $x^{\star}$ across all NE. This combined with individual rationality Lemma 4.8, gives us Theorem 4.1. 


\section{A Mechanism with Strong Budget Balance}

We now turn our attention to problem $\left(\mathrm{CP}_{2}\right)$. So in this case we have the agents in $\mathcal{N}$, who are the owners and users that wish to allocate the good amongst themselves in a way that maximises $\sum_{k i \in \mathcal{N}} u_{k i}$. In this case one can now think of taxes as a way of facilitating efficient redistribution of the already available good. Since all payments are made amongst agents in $\mathcal{N}$ and we have quasi-linear utilities, this clearly tells us that $\sum_{k i \in \mathcal{N}} t_{k i}$ must be zero. This interpretation is slightly different from Section 4, where taxes were indeed payments made to the seller for provisioning of the good.

All of the above is required to be done again under the assumption of strategic agents, which means the designer (who is a third party) still has the problem of information elicitation and moreover has to make sure that the wealth has to be redistributed in a way that we still get $x^{\star}$ allocation at the all equilibria. Here we will say that the mechanism fully implements maximising social welfare allocation if in addition to the previous conditions, we also have SBB.

\subsection{Information assumptions. These are the same as Section 4.}

For creating a mechanism in this formulation, main difference with the previous section, is that we have to find a way of redistributing the total tax paid by all the agents. In the last section we saw that the total payment made at the equilibrium is

$$
B=\sum_{k i \in \mathcal{N}}\left(x_{k i} \sum_{l \in \mathcal{L}_{k i}} \alpha_{k i}^{l} p_{k, i}^{l}\right)=r \sum_{k i \in \mathcal{N}}\left(y_{k i} \sum_{l \in \mathcal{L}_{k i}} \alpha_{k i}^{l} p_{k, i}^{l}\right)
$$

since all other tax terms were zero at equilibrium. We will redistribute taxes by modifying tax function for each agent only using messages from other agents. This has the advantage of keeping our equilibrium calculations in line with Section 4, since deviations by an agent wouldn't affect his utility through this additional term. In view of this, we can express $B$ as follows

$$
B=r \sum_{k i \in \mathcal{N}}\left(\sum_{l \in \mathcal{L}_{k i}} \frac{1}{N^{l}-1} \sum_{k^{\prime} j \in \mathcal{N}^{l} \backslash\{k i\}} \alpha_{k^{\prime} j}^{l} p_{k^{\prime}, j}^{l} y_{k^{\prime} j}\right),
$$

where each term of the outer summation depends only on demands of agents other than the $k i^{\text {th }}$ one. This means that each term in the parenthesis (scaled by the factor $r$ ) can now be used as the desired additional tax for user ki. Observe, however, that in our mechanism, each agent's demand affects the factor $r$ as well. So, if all agents can agree on value of $r$ then we can use that signal to create the term that facilitates budget balance.

In lieu of this, our mechanism here works by asking for an additional signal $\rho_{k i}$ from every agent and imposing an additional tax of $\left(\rho_{k i}-r\right)^{2}$, thereby essentially ensuring that all agents agree on the value of $r$ (via $\rho_{k i}$ 's) at equilibrium. Finally, we use $\bar{\rho}_{-k i}$ (cf. (19)) as a proxy for $r$ in (15) - somewhat similar to what we did with $\bar{w}_{-k}^{l}$ 's. 
5.2. Mechanism. Now the actions sets $\mathcal{S}_{k i}$ for agents will be $\mathbb{R}_{+} \times \mathbb{R}_{+}^{2 L_{k i}} \times \mathbb{R}_{+}$and actions will look like $s_{k i}=\left(y_{k i}, p_{k i}, \rho_{k i}\right)$.

The designer announces the contract $h: \mathcal{S} \rightarrow \mathbb{R}_{+}^{N} \times \mathbb{R}^{N}$ and asks each agent to submit their message $\left(y_{k i}, p_{k i}, \rho_{k i}\right)$. Then he makes allocations and taxes based on the contract for each agent $k i \in \mathcal{N}$ exactly as in (WBB) case, with the only exception that the tax is now defined as

$$
h_{t, k i}(s)=t_{k i}=\sum_{l \in \mathcal{L}_{k i}} t_{k i}^{l}+\left(\rho_{k i}-r\right)^{2}
$$

if $G_{k}^{l} \geq 2$, then again using agents $k j$ and $k e$ as described after (10), we have

$$
\begin{aligned}
t_{k i}^{l}= & x_{k i} \alpha_{k i}^{l} q_{k j}^{l}+\left(q_{k i}^{l}-p_{k e}^{l}\right)^{2}+\left(w_{k}^{l}-\bar{w}_{-k}^{l}\right)^{2}+q_{k j}^{l}\left(p_{k i}^{l}-q_{k j}^{l}\right)\left(m_{k}^{l}-\alpha_{k i}^{l} x_{k i}\right) \\
& +\bar{w}_{-k}^{l}\left(w_{k}^{l}-\bar{w}_{-k}^{l}\right)\left(c^{l}-\sum_{k^{\prime} \in \mathcal{K}^{l}} m_{k^{\prime}}^{l}\right)-\frac{\bar{\rho}_{-k i}}{N^{l}-1} \sum_{k^{\prime} j \in \mathcal{N}^{l} \backslash\{k i\}} \alpha_{k^{\prime} j}^{l} q_{k^{\prime} j^{\prime}}^{l} y_{k^{\prime} j}
\end{aligned}
$$

where agent $k^{\prime} j^{\prime}$ is an agent who has alternate representation $g_{k^{\prime}}^{l}(j)-1$ on link $l$, if $G_{k^{\prime}}^{l} \geq 2$. However if $G_{k^{\prime}}^{l}=1$ we would use $\bar{w}_{-k^{\prime}}^{l}$ instead of $q_{k^{\prime} j^{\prime}}^{l}$.

Similarly for $G_{k}^{l}=1$, we have

$$
\begin{aligned}
& t_{k i}^{l}=x_{k i} \alpha_{k i}^{l} \bar{w}_{-k}^{l}+\left(w_{k}^{l}-\bar{w}_{-k}^{l}\right)^{2}+\bar{w}_{-k}^{l}\left(p_{k i}^{l}-\bar{w}_{-k}^{l}\right)\left(m_{k}^{l}-\alpha_{k i}^{l} x_{k i}\right) \\
& +\bar{w}_{-k}^{l}\left(w_{k}^{l}-\bar{w}_{-k}^{l}\right)\left(c^{l}-\sum_{k^{\prime} \in \mathcal{K}^{l}} m_{k^{\prime}}^{l}\right)-\frac{\bar{\rho}_{-k i}}{N^{l}-1} \sum_{k^{\prime} j \in \mathcal{N}^{l} \backslash\{k i\}} \alpha_{k^{\prime} j}^{l} q_{k^{\prime} j^{\prime}}^{l} y_{k^{\prime} j}
\end{aligned}
$$

In addition to previous definitions, here (for both equations) we have

$$
\bar{\rho}_{-k i}:=\frac{1}{N-1} \sum_{k^{\prime} j \in \mathcal{N} \backslash\{k i\}} \rho_{k^{\prime} j}
$$

Here we will call the corresponding game $\mathfrak{G}_{0}$, for which utilities will be

$$
\hat{u}_{k i}(s)=v_{k i}\left(x_{k i}\right)-t_{k i}=v_{k i}\left(h_{x, k i}(s)\right)-h_{t, k i}(s) \quad \forall k i \in \mathcal{N}
$$

We will now move on to results section and discuss the implications of the modifications there.

5.3. Results. With this new mechanism, we will again have full implementation (note that for individual rationality there is no seller here). The only term in $\hat{u}_{k i}$ that is affected by $\rho_{k i}$ is $-\left(\rho_{k i}-r\right)^{2}$, so all the Lemmas from Section 4 will go through with minor modifications and we will have our main result using the same line of argument as for Theorem 4.1. Note here that, terms in $\hat{u}_{k i}$ affected by $p_{k i}^{l}, q_{k i}^{l}$ 's are the same as before but for $y_{k i}$ there is a new term $-\left(\rho_{k i}-r\right)^{2}$ which is affected by it.

THEOREM 5.1 (Full Implementation). For game $\mathfrak{G}_{0}$, there is a unique allocation, $x$, corresponding to all NE. Moreover, $x=x^{\star}$, the maximiser of (CP), where individual rationality is satisfied for all agents in $\mathcal{N}$. 
In addition to all the properties from Section 4, here we will characterise $\rho_{k i}$ 's at equilibrium and then go on to show SBB at equilibrium.

Instead of proving the results from Section 4 for this new mechanism, we will outline their proofs and only show the rigorous proofs for new properties.

- Primal Feasibility - Since allocation function is the same as before, this result holds here as well.

- Equal Prices at equilibrium - This was proved by taking price deviations only and keeping other parameters of the signal constant, so the same argument works here as well (noting that no new price related terms have been added in the new mechanism).

Before moving on to other results, we will show common $\rho_{k i}$ 's at equilibrium.

Lemma 5.2. At any $N E s=(y, P, \rho)$ of game $\mathfrak{G}_{0}$, we have $\rho_{k i}=r \quad \forall k i \in \mathcal{N}$.

ProOf. Suppose not, i.e. assume $\exists k j \in \mathcal{N}$ such that $\rho_{k j} \neq r$. In this case agent $k j$ can deviate with only changing $\rho_{k j}^{\prime}=r$ (which also means $r$ is the same as before deviation, since demand $y$ doesn't change). It's easy to see that this is a profitable deviation, since change in utility of agent $k j$ will be only through the term involving $\rho_{k j}$.

$$
\Delta \hat{u}_{k j}=-\left(\rho_{k j}^{\prime}-r\right)^{2}+\left(\rho_{k j}-r\right)^{2}=\left(\rho_{k j}-r\right)^{2}>0
$$

Note however that although $\rho_{k i}$ are same for all $k i$ at any equilibrium, that common value, $r$, will be different across equilibria. This is obvious since magnitude of vector $y$ changes across equilibria.

Now we move on with properties from Section 4.

- Dual Feasibility - This is obvious here as well.

- Complimentary Slackness - This was proved by taking only price deviations and hence the same argument works here as well.

- Stationarity - Now the additional term in the derivative here will be

$$
\left.\frac{\partial \hat{u}_{k i}}{\partial y_{k i}^{\prime}}\right|_{\text {new }}=\underbrace{\left.\frac{\partial \hat{u}_{k i}}{\partial y_{k i}^{\prime}}\right|_{o l d}}_{T_{1}}-\underbrace{2\left(\rho_{k i}^{\prime}-r^{\prime}\right)\left(-\frac{\partial r^{\prime}}{\partial y_{k i}^{\prime}}\right)}_{T_{2}}
$$

So we claim as before that if $T_{1}$ is positive, we can increase $y_{k i}^{\prime}$ from $y_{k i}$ to be better-off. Here however we would have to make sure that agent $k i$ deviates with $\rho_{k i}^{\prime}$ simultaneously to make it equal to $r^{\prime}$, so that the contribution of the $T_{2}$ term to the derivative is zero. The only thing left to notice here is that the change in $\rho_{k i}^{\prime}$ is such that not only the term $T_{2}$ is zero but also that the contribution of term $-\left(\rho_{k i}^{\prime}-r^{\prime}\right)^{2}$ to the utility is zero before and after deviation - so this deviation doesn't change other partial derivatives. 
Similar argument also works when $T_{1}$ is negative and we get the stationarity property here as well.

With this we will have unique allocation at every equilibria, since solution to KKT is unique (as far as allocation is concerned). This unique allocation will be $x^{\star}$; also the prices will be $\lambda^{\star}$, same as before.

Now we verify the existence of equilibria. The arguments here will be similar to the ones in the proof of Lemma 4.7. First order conditions can again be shown to be satisfied, the only difference is that here we will also use $\rho_{i}=r$ at equilibrium. The Hessian $H$ for any agent $k i$ here will be of order $\left(2 L_{k i}+2\right) \times\left(2 L_{k i}+2\right)$ where 1 st, 2 nd row and column represent $y_{k i}$, $\rho_{k i}$ respectively whereas the remaining rows and columns represent $p_{k i}^{l}$ 's and $q_{k i}^{l}$ 's. The generic matrix $A_{0}$ for $H$ will then be of the form

$$
A_{0}=\left[\begin{array}{ll}
A & 0 \\
0 & D
\end{array}\right]
$$

where $D=(-2) I_{L_{k i}}$ and matrix $A$, of order $\left(L_{k i}+2\right) \times\left(L_{k i}+2\right)$, will have elements

$$
\begin{gathered}
a_{11}=-\frac{a}{|y|}-\frac{d}{|y|^{4}} \quad a_{12}=a_{21}=-\frac{e}{|y|^{2}} \quad a_{i j}=a_{j i}=0 \quad \forall \quad i, j>1, i \neq j \\
a_{22}=-2 \quad a_{i i}=-2 \quad a_{1 i}=a_{i 1}=\frac{b_{i-1}}{|y|} \quad \forall \quad 3 \leq i \leq L_{k i}+2
\end{gathered}
$$

where $a, d, e>0$. As before, all we have to is show that all eigenvalues of $A$ are negative (since that is clearly true for $D$ ). Writing the characteristic equation we will again get that -2 is a repeated eigenvalue, $L_{k i}$ times. And the equation for remaining two roots is

$$
\lambda^{2}+\lambda\left(2+\frac{a}{|y|}+\frac{d}{|y|^{4}}\right)+\left(\frac{2 a}{|y|}+\frac{2 d}{|y|^{4}}+\frac{C}{|y|^{2}}-\frac{E}{|y|^{4}}\right)=0
$$

Necessary and sufficient conditions for the roots of above quadratic to be negative are again that coefficient of $\lambda$ and the constant term are both positive. Coefficient of $\lambda$ is clearly positive, and for $|y|$ big enough the constant term also becomes positive, irrespective of signs of $C$ and $E$. Hence here also we get NE for all $y$ (along a fixed direction) such that $|y|$ is large enough.

- Individual Rationality - This is obvious in here because we are only redistributing money from the previous case, so if the mechanism there was individually rational it will be here too.

LEMma 5.3 (Strong Budget Balance). At any $N E s=(y, P, \rho)$ of game $\mathfrak{G}_{0}$, with corresponding taxes $\left\{t_{k i}\right\}_{k i \in \mathcal{N}}$, we have $\sum_{k i \in \mathcal{N}} t_{k i}=0$. 
Proof. We know that terms 2, 3, 4 and 5 in (17) and (18) are zero at equilibrium and so we can write (at equilibrium)

$$
\begin{aligned}
& \sum_{k i \in \mathcal{N}} t_{k i}=\sum_{k i \in \mathcal{N}} x_{k i}\left(\sum_{l \in \mathcal{L}_{k i}} \alpha_{k i}^{l} p_{k, i}^{l}\right)-r \sum_{l \in \mathcal{L}_{k i}} \frac{1}{N^{l}-1} \sum_{k^{\prime} j^{\prime} \in \mathcal{N}^{l} \backslash\{k i\}} \alpha_{k^{\prime} j^{\prime}}^{l} p_{k^{\prime}, j^{\prime}}^{l} y_{k^{\prime} j^{\prime}} \\
& \Rightarrow \quad \sum_{k i \in \mathcal{N}} t_{k i}=\sum_{k i \in \mathcal{N}} \sum_{l \in \mathcal{L}_{k i}}\left(x_{k i} \alpha_{k i}^{l} p_{k i}^{l}-\frac{1}{N^{l}-1} \sum_{k^{\prime} j^{\prime} \in \mathcal{N}^{l} \backslash\{k i\}} \alpha_{k^{\prime} j^{\prime}}^{l} p_{k^{\prime}, j^{\prime}}^{l} x_{k^{\prime} j^{\prime}}\right)
\end{aligned}
$$

Consider the coefficient of $x_{k i}$ for any agent $k i$ in the above expression

$$
\begin{aligned}
\sum_{l \in \mathcal{L}_{k i}}\left(\alpha_{k i}^{l} p_{k, i}^{l}-\frac{1}{N^{l}-1} \sum_{k^{\prime} j^{\prime} \in \mathcal{N}^{l} \backslash\{k i\}} \alpha_{k i}^{l} p_{k, i}^{l}\right) & =\sum_{l \in \mathcal{L}_{k i}}\left(\alpha_{k i}^{l} p_{k, i}^{l}-\frac{1}{N^{l}-1}\left(N^{l}-1\right) \alpha_{k i}^{l} p_{k, i}^{l}\right) \\
& =0,
\end{aligned}
$$

which proves the claim.

Proof of Theorem 5.1. So by the preceding properties, we get allocation $x^{\star}$, prices $\lambda^{\star}$ at all equilibria. Then SBB and individual rationality give us the desired full implementation.

\section{Discussion and Generalizations}

Relevant Literature. The problem considered in [9] is essentially equivalent to ours (with relaxed assumptions (A3)-(A4), the additional property of SBB on and off equilibrium, and feasibility only at equilibrium). In the following we point out two problems with the mechanism described in [9], the first of which has been addressed by the authors in a correction in [10].

The first problem is that the claim of efficiency made in [9] is not valid. In particular the proof of [9, Theorem 6] is incorrect: the utilities need not have zero derivatives at equilibrium, since they are discontinuous at equilibrium and the only allowable deviations of $x_{k i}$ are downwards deviations. Intuitively, this problem arises due to using hard constraints for ensuring feasibility of allocation: when the demanded allocation is not feasible, a large penalty is imposed on the agents. This approach creates discontinuities of the utility functions at the boundaries of the achievable region and thus renders invalid any attempt to link the corresponding NE with the KKT conditions of the corresponding centralized problem. The authors of [9] have suggested a modified mechanism in [10] for overcoming this problem. This modification is similar in spirit to the correction [8] for the problem of mechanism design for unicast service [7].

The second problem with the mechanism in [9] which persists in the correction [10], is that the claim of existence of NE made in [9, Theorem 1] is incorrect, resulting in a mechanism that does not always have pure-strategy NE. In particular, the mechanism in [9], makes allocations according to demand i.e. $x_{k i}=y_{k i}$ and sets beforehand the price paid by agent $k i$ on link $l$ as 0 if $k i \notin \arg \max _{i \in \mathcal{G}_{k}^{l}}\left\{\alpha_{k j}^{l} x_{k j}\right\}$. This is in line with KKT conditions; complimentary slackness 
indeed requires $\mu_{k i}^{l}{ }^{\star}\left(\alpha_{k i}^{l} x_{k i}^{\star}-m_{k}^{l \star}\right)=0$, so the price for agent $k i$ (as above) should be 0 . The problem however is that in some cases an agent $k i$ could profitably deviate from the stated NE by reducing demand $x_{k i}$ by an arbitrary small amount $\epsilon$. This would ensure agent $k i$ no longer belongs to $\arg \max _{i \in \mathcal{G}_{k}^{l}}\left\{\alpha_{k j}^{l} x_{k j}\right\}$ on those links where $\arg \max _{i \in \mathcal{G}_{k}^{l}}\left\{\alpha_{k j}^{l} x_{k j}\right\}$ had more than one agent and $k i$ was one of them. This is profitable because the tax would reduce in a discontinuous manner whereas the reduction in utility from reduced allocation would be continuous (since $v_{k i}$ is continuous). A similar situation arises in the correction [10], although taxes there are not fixed at zero ${ }^{4}$ for agents not belonging to $\arg \max _{i \in \mathcal{G}_{k}^{l}}\left\{\alpha_{k j}^{l} x_{k j}\right\}$. Intuitively, this problem arises by the attempt to hard-wire the solutions implied by the KKT conditions into the mechanism.

Indeed, one of the contributions of our work in this paper is embedding of the constraints within the mechanism in an implicit way, such that the allocations are always feasible (on and off equilibrium), and are continuous and (piecewise) differentiable with respect to the demands. In addition, the KKT conditions are implicitly embedded in the mechanism by the use of the additional complimentary slackness terms.

Strong Budget Balance off-equilibrium. In this work, we do not view SBB off equilibrium as an important property of a mechanism. However, in the following we sketch a modification of the proposed mechanism that results in arbitrarily close to SBB off equilibrium. In Section 5, we use $\bar{\rho}_{-k i}$ 's simply as a way to get SBB at equilibrium. Here $\bar{\rho}_{-k i}$ was used as a proxy for $r$, since we knew that at equilibrium we will have $\bar{\rho}_{-k i}=r$. We could, in addition to this, also use $\bar{\rho}_{-k i}$ as a proxy for $r$ in the allocation i.e. $x_{k i}=\bar{\rho}_{-k i} y_{k i}$. Although we won't have feasibility of allocation off-equilibrium, this will ensure that the first term (payment) and the sixth term (redistribution) in the tax function (refer to (17)) cancel out when we sum over all agents - on or off-equilibrium. This will give us something close to SBB at all points in the message space $\mathcal{S}$ and not just at equilibria - for this all we have to notice is that in (16) we could introduce any positive constant in front of terms 2, 3, 4 and 5 and all the results would still go through. So by making that constant small enough we could restrict the contribution of those terms to $\sum_{k i \in \mathcal{N}} t_{k i}$, which we couldn't do with terms 1 and 6 since term 1 compares with $v_{k i}$, for which we do not know the scaling and term 6 is introduced to cancel out term 1 when we sum over all agents.

Using Nash equilibrium as solution concept. As mentioned before, Nash equilibrium as a solution concept applies to complete information games. Our motivation to use mechanism design, implementation to be specific, for resource allocation on the Internet lied in the fact that the designer (or equivalently a centralised authority responsible for enforcing contracts and overlooking allocations) did not have the private information of Internet agents. So to assume that the players themselves have complete knowledge about each others' private information might be considered an impractical assumption. One justification used is that the mechanism is

\footnotetext{
${ }^{4}$ It is actually unclear what is the meaning of [10, eq. (2)], since user $k$ is defined only in the group of users with maximum demand.
} 
designed prior to agents' realising their private information, just like how a constitution designed a long time ago is still used years later. So the designer cannot know the private information of agents in advance. This in conjunction with the assumption that agents involved have a lot to lose or gain from the allocation (hence have an incentive to learn about private information others' separately) may form one instance of an Internet system where Nash equilibrium is a valid solution concept. Another justification is by interpreting Nash equilibria as the outcome of a dynamic adjustment process, where players eventually learn enough about each other to converge to the Nash equilibrium action. Readers may refer to [24] for an exposition on the above mentioned dynamic adjustment argument and to [25] for learning methods used in game theoretic setup.

Future research directions. We believe further investigation is needed into this problem. A very important research direction would be to look into the possible outcomes from a Bayesian framework and/or designing a new mechanism appropriate for this framework. One could also look into informational robustness of this mechanism itself.

\section{References}

[1] M. O. Jackson, "A crash course in implementation theory," Social choice and welfare, vol. 18, no. 4, pp. 655-708, 2001.

[2] D. Garg, Y. Narahari, and S. Gujar, "Foundations of mechanism design: A tutorial part 1-key concepts and classical results," in Sadhana (Academy Proceedings in Engineering Sciences), vol. 33, no. 2. Indian Academy of Sciences, 2008, pp. 83-130.

[3] - " "Foundations of mechanism design: A tutorial part 2-advanced concepts and results," Sadhana, vol. 33, no. 2, pp. 131-174, 2008.

[4] R. T. Maheswaran and T. Bașar, "Nash equilibrium and decentralized negotiation in auctioning divisible resources," Group Decision and Negotiation, vol. 12, no. 5, pp. 361-395, 2003.

[5] R. T. Maheswaran and T. Basar, "Social welfare of selfish agents: motivating efficiency for divisible resources," in Decision and Control, 2004. CDC. 43rd IEEE Conference on, vol. 2. IEEE, 2004, pp. 1550-1555.

[6] S. Yang and B. Hajek, "Revenue and stability of a mechanism for efficient allocation of a divisible good," preprint, 2005.

[7] A. Kakhbod and D. Teneketzis, "An efficient game form for unicast service provisioning," Automatic Control, IEEE Transactions on, vol. 57, no. 2, pp. 392-404, 2012.

[8] —_, "Correction to "An efficient game form for unicast service provisioning", , 2013. [Online]. Available: http://arxiv.org/abs/0910.5502

[9] - "An efficient game form for multi-rate multicast service provisioning," Selected Areas in Communications, IEEE Journal on, vol. 30, no. 11, pp. 2093-2104, 2012.

[10] - "Correction to "An efficient game form for multi-rate multicast service provisioning", " Selected Areas in Communications, IEEE Journal on, vol. 31, no. 7, pp. 1355-1356, 2013.

[11] D. Rubenstein, J. Kurose, and D. Towsley, "The impact of multicast layering on network fairness," in $A C M$ SIGCOMM Computer Communication Review, vol. 29, no. 4. ACM, 1999, pp. 27-38.

[12] E. E. Graves, R. Srikant, and D. Towsley, Decentralized computation of weighted max-min fair bandwidth allocation in networks with multicast flows. Springer, 2001. 
[13] S. Sarkar and L. Tassiulas, "Fair allocation of utilities in multirate multicast networks," in Proc. Allerton Conf. Commun., Control, Comp., vol. 37, 1999, pp. 718-727.

[14] — , "Fair allocation of utilities in multirate multicast networks: a framework for unifying diverse fairness objectives," Automatic Control, IEEE Transactions on, vol. 47, no. 6, pp. 931-944, 2002.

[15] K. Kar and L. Tassiulas, "Layered multicast rate control based on lagrangian relaxation and dynamic programming," Selected Areas in Communications, IEEE Journal on, vol. 24, no. 8, pp. 1464-1474, 2006.

[16] K. Kar, S. Sarkar, and L. Tassiulas, "Optimization based rate control for multirate multicast sessions," in INFOCOM 2001. Twentieth Annual Joint Conference of the IEEE Computer and Communications Societies. Proceedings. IEEE, vol. 1. IEEE, 2001, pp. 123-132.

[17] F. P. Kelly, A. K. Maulloo, and D. K. Tan, "Rate control for communication networks: shadow prices, proportional fairness and stability," Journal of the Operational Research society, vol. 49, no. 3, pp. 237-252, 1998.

[18] A. Sinha and A. Anastasopoulos, "Mechanism design for unicast service on the internet," University of Michigan, Tech. Rep., 2013. [Online]. Available: http://www.eecs.umich.edu/techreports/systems/cspl/ cspl-414.pdf

[19] R. Johari and J. N. Tsitsiklis, "Communication requirements of VCG-like mechanisms in convex environments," in Proceedings of the Allerton Conference on Control, Communications and Computing, 2005.

[20] S. Yang and B. Hajek, "VCG-Kelly mechanisms for allocation of divisible goods: Adapting VCG mechanisms to one-dimensional signals," Selected Areas in Communications, IEEE Journal on, vol. 25, no. 6, pp. 12371243, 2007.

[21] V. Krishna, Auction theory. Academic press, 2009.

[22] S. Reichelstein and S. Reiter, "Game forms with minimal message spaces," Econometrica: Journal of the Econometric Society, pp. 661-692, 1988.

[23] T. Groves and J. Ledyard, "Optimal allocation of public goods: A solution to the" free rider" problem," Econometrica: Journal of the Econometric Society, pp. 783-809, 1977.

[24] H. P. Young, Individual strategy and social structure: An evolutionary theory of institutions. Princeton University Press, 2001.

[25] D. Fudenberg, The theory of learning in games. MIT press, 1998, vol. 2. 